\title{
Improving SEVIRI-Based Hotspots Detection by Using Multiple Simultaneous Observations
}

\author{
Giovanni Laneve (i), Member, IEEE, Giancarlo Santilli, and Roberto Luciani (i)
}

\begin{abstract}
Geostationary satellites like meteosat second generation (MSG) allow the detection and monitoring of thermal anomalies (wild fires and volcanic eruptions) with a refresh frequency ranging from 5 to $15 \mathrm{~min}$. Such a frequency meets the requirements of the institutions involved in monitoring and containing the fire events and could provide information on the temporal behavior of the fire (through fire radiative power) and the spatial distribution of the events with the related hazard for the population and infrastructure when more occurrences are simultaneously present. A limitation of the operational applicability of this tool is currently represented by the low spatial resolution of the MSG/SEVIRI sensor ranging from $3 \mathrm{~km}$ at the equator to $4.5 \mathrm{~km}$ at Mediterranean latitudes. The limitations related to the sensitivity of the geostationary sensor to fire sizes have been, at least in part, overcome by introducing specific algorithms. However, the reduced accuracy in the geographic localization of the fire, which can, in principle, occupy any position in an area of about $16 \mathrm{~km}^{2}$ (at Mediterranean latitudes), makes this information not very interesting for the institutions involved in firefighting. This paper analyzes the feasibility of improving the localization of the thermal anomalies (hotspots) by combining images acquired simultaneously from different MSG satellites located at different longitudes. In particular, we combine the images acquired by MSG-9 located at long. 9.0 ${ }^{\circ}$, MSG-10 located at $0.0^{\circ}$ and MSG-8 located at long. $41.5^{\circ}$. The results confirm the possibility of improving the accuracy of the detection by exploiting the observation of the events from different positions in space.
\end{abstract}

Index Terms-Geostationary, localization, SEVIRI, wildfire.

\section{INTRODUCTION}

$\mathbf{E}$ ARLY detection of forest fires based on satellite images presents two main justifications.

1) It could help reduce the response time of the reaction system. The possibility of realizing a timely detection of a fire requires a quasi-continuous observation of the area of interest. This can be obtained by means of a single geostationary satellite [such as the geostationary operational environmental satellites (GOES) or meteosat second generation (MSG)] or by means of a constellation made up of

Manuscript received September 29, 2018; revised January 8, 2019; accepted January 31, 2019. (Corresponding author: Giovanni Laneve.)

G. Laneve and R. Luciani are with the School of Aerospace Engineering, Sapienza University of Rome, Rome 00185, Italy (e-mail: giovanni. laneve@uniroma1.it; roberto.luciani@uniroma1.it).

G. Santilli is with the UnB Gama Faculty (FGA), University of Brasilia, Brasilia 70910-900, Brazil (e-mail: santilli@ aerospace.unb.br).

Color versions of one or more of the figures in this paper are available online at http://ieeexplore.ieee.org.

Digital Object Identifier 10.1109/JSTARS.2019.2898126 a high number (at least 12) of satellites in low earth orbit (LEO).

2) In case of several fires occurring at the same time, it provides an unbiased tool for the decision makers to optimize the use of, often, limited resources.

In the last few decades, the occurrences of fires in the European Mediterranean countries (France, Greece, Italy, Portugal, and Spain) have increased from an average of 40000 fires per year in the period 1970-1980, corresponding to a forested area of about 228000 ha/year, to about 50000 fires and 470000 burned hectares in the last decade [1].

According to the fire-fighting agencies, a satellite-based fire detection system can be considered operationally useful for Mediterranean countries when fires with a minimum extension of $1500 \mathrm{~m}^{2}$ can be detected with a temporal resolution of $30 \mathrm{~min}$. In fact, such a system must be able to detect fires in their early stages, when it is possible to extinguish them more easily. The use of satellite images to detect forest fires is a well-known application based on the exploitation of different sensors [such as the advanced very high resolution radiometer (AVHRR), the terra moderate resolution imaging spectroradiometer (MODIS), GOES, etc.] characterized by various spatial resolutions [2]-[9]. At present, several web services providing active fire information derived from satellite images are available [fire information for resource management system (FIRMS), European forest fire information system (EFFIS), fire identification mapping and monitoring algorithm (FIMMA), wildfire automated biomass burning algorithm (ABBA), etc.]. However, it must be pointed out that most of these applications do not aim at supporting counteracting operations and event management. In fact, the main purpose of fire-detection applications (except in very rare cases) is that of carrying out a statistical study of the events and their possible environmental impact in terms of burnt area and variation of the optical characteristics of the atmosphere due to burning products (global-scale climate change) [6]. In particular, because of the sensors' reduced spatial resolution, applications based on geostationary sensors (GOES, MSG) were mostly devoted to better estimate the aerosol emission of forest fires, which contribute significantly to the global greenhouse effect.

Fires occurring in the Mediterranean area are rarely significant in terms of burning products released into the atmosphere. Nevertheless, they have a dramatic impact on the extension of vegetated areas in regions with relatively scarce vegetation and on human lives and infrastructure.

Several research projects aiming at realizing early warning systems based on remote sensing have been developed in recent 
years (AFIS, EFFIS, MACC, SIGRI, AF3, etc.). However, they all face the current technological limitations of satellite sensors in providing suitable spatial and/or temporal resolutions.

Algorithms specifically devoted to fire detection using lowresolution multispectral imagery can be categorized in two classes: Fixed threshold and contextual threshold [4]-[11]. Both methods can be based on a single- or multichannel approach and show some limits related to the selection of the thresholds to detect fire or potential fire pixels. In fact, the fixed threshold technique gives good results for a particular region/biomass [6]. On the other hand, the contextual technique is applicable to a wide range of conditions, but it requires conservative thresholds in the attempt to reduce false fire detection [6]. To overcome these limits, in the framework of a project funded by the Italian Space Agency a third approach, able to exploit the high sampling frequency of the MSG/SEVIRI sensor and potentially capable of supporting the Italian Fire Brigades in the firefighting phase, was selected. It is based on a change-detection technique to maximize the fire-recognition capabilities of the system in spite of its limited spatial resolution [12], [13]. This technique consists of comparing two or more consecutive images acquired at 15 -min (or $5 \mathrm{~min}$ ) intervals, for which any detected thermal change can be attributed to fast dynamic phenomena, such as fires, when natural changes are modeled and removed.

However, apart from the sensitivity of the detection algorithm, another significant limit of the information on forest fires provided by geostationary satellites concerns the low spatial resolution of the images (about $4 \mathrm{~km}$ at Italian latitudes). This means that the thermal anomaly can be geolocalized with an approximation of $\pm 2 \mathrm{~km}$. In some cases, this approximation increases the skepticism of the users concerning this tool even if the advantage of an instrument capable of providing a synoptic view of fire distribution at national level and an objective way to assess their intensity [through the fire radiative power] remains. Therefore, the present paper aims at presenting the results obtained in the process of improving the accuracy of the localization of forest fires detected by geostationary satellites by combining the images acquired simultaneously from two or more MSG satellites (see Fig. 1). In particular, Section II is devoted to describing the data used and the method applied, while Section III is devoted to describing and discussing the results of the adopted approach.

\section{DATA AND METHOD}

The area of interest is represented by the whole Italian country. In Fig. 2, two of the Italian regions most affected by fires during the summer season are shown. These are Calabria (right) and Sardinia (left). As an example, Fig. 2 also shows the fire events, as provided by National Fire Corps, that occurred in Calabria and Sardinia from the July 20 to the August 20,2017. These data can be used to show the incidence of the wildfires phenomenon in the regions, but unfortunately, they cannot be used, in general, to check the accuracy of the hotspot localization based on satellite images (geostationary and polar sensors: MSG/SEVIRI, Terra and Aqua/MODIS and SNPP/VIIRS). Sometimes, in fact, the geolocation of the event refers to the municipality where it

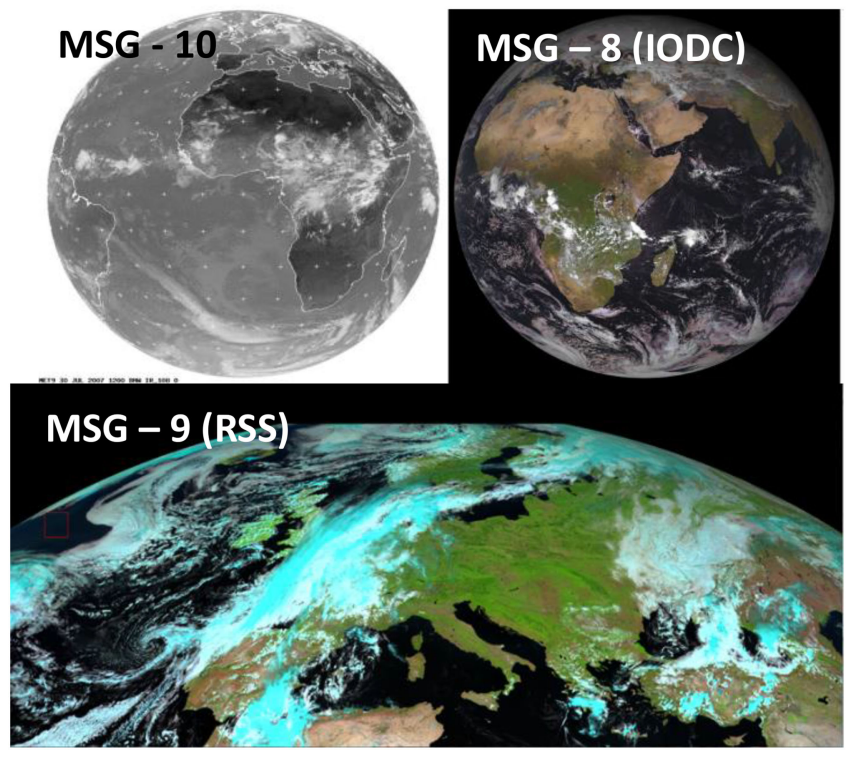

Fig. 1. Example of images acquired by the SEVIRI sensors on board of the three MSG satellites.

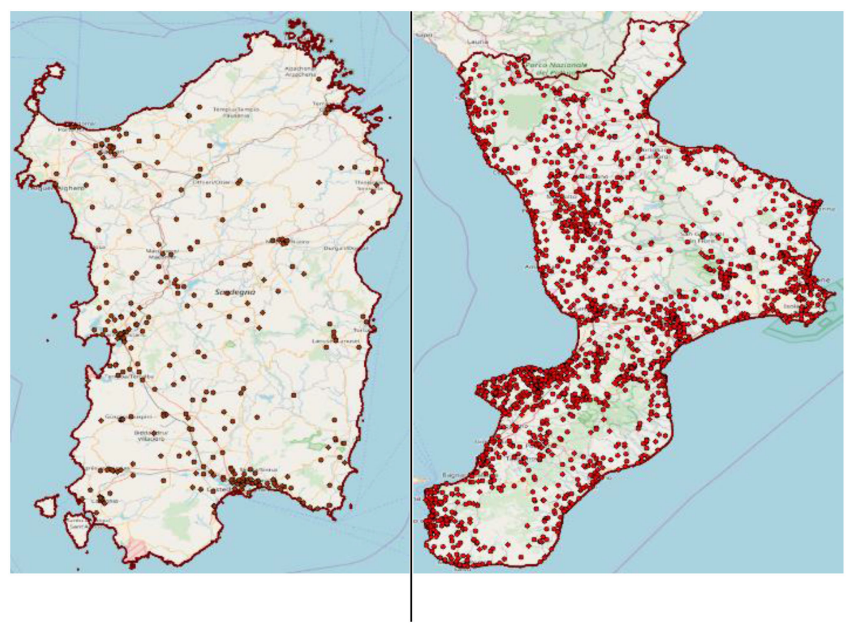

Fig. 2. Distribution of the fire events occurred in Calabria (right) and Sardinia (left) in the period July 20-August 20, 2017 (Courtesy of National Fire Brigades).

occurred or to the first intervention point reached by intervention teams. Therefore, the analysis was done first by comparing SEVIRI hotspots with those provided by MODIS and visible infrared imaging radiometer suite (VIIRS), and additionally by using the ground-based measurements of the events for which the location accuracy had been assessed through the corresponding burned areas. Concerning the satellite data, MODIS and VIIRS hotspots were obtained from the FIRMS Web Fire Mapper. SEVIRI hotspots were computed by applying the SFIDE software [12], [13], developed by the author and colleagues, to the MSG-9 RSS (rapid scanning service, Fig. 1), which provides images with 5 min refresh frequency and the MSG-8 IODC (Indian Ocean data coverage, Fig. 1), which provides images with 15 min refresh frequency. The SFIDE algorithm was originally developed in 2005 [12], since then it has been 
significantly improved exploiting international funds, cooperation of Regional Civil Protection Departments and Ph.D. students [13]. The algorithm exploits the high refresh frequency of the SEVIRI images to detect the smallest possible changes in the averaged brightness temperature of a pixel. The images of the MSG satellite series are directly received at the University laboratory (EOSIAL, eosial.psm.uniroma1.it) through the EUMETCAST dissemination system of EUMETSAT. As already recalled earlier due to the spatial resolution of the SEVIRI sensor at our latitude (about $4 \mathrm{~km}$ ), in general, the position of the fire in the pixel can be defined with an approximation as high as $\pm 2 \mathrm{~km}$.

The analysis also involved the use of the hotspots directly provided by EUMETSAT for MSG-HRIT (high rate information transmission) and MSG-IODC [14], [15].

In order to improve the accuracy of the geolocation of the hotspot within the pixel, we propose a method composed of two steps.

1) Combining the images acquired simultaneously by the SEVIRI sensor on board of MSG 9 RSS (located at $9.5^{\circ}$ longitude) with those provided by MSG-8 IODC (located at $41.5^{\circ}$ longitude), Fig. 1 . The comparison can be made every 15 min since MSG-8 has a refresh frequency of 15 min.

2) Superimpose the SEVIRI image on a higher resolution vegetation fuel map of the area of interest in order to associate the hotspot position (corresponding to the pixel center coordinates) to a probability distribution of the fire position based on the characteristics of the vegetation in the $4 \times 4 \mathrm{~km}^{2}$ pixel. The $250 \mathrm{~m}$ spatial resolution fuel map used here has been computed by JRC for the whole Europe in the framework of the FUELMAP project.

The idea of exploiting the images acquired simultaneously from the RSS and IODC sensors which observe the Earth surface from different positions in space is based on the following consideration. The pixel grid corresponding to the image acquired by each sensor has a different distribution (see Fig. 3). As a consequence, the actual position of the fire in the pixel of RSS defines the pixel of the IODC image, in which the same thermal anomaly is detected. Fig. 3 shows the pixels grid corresponding to the image of a portion of the area of interest acquired by RSS. In the same figure, on the right, zooming in the area of interest it is possible to notice the difference in the pixels grid of the images taken by the two different satellites. Fig. 3, bottom, shows the RSS pixels grid in green and the IODC pixels grid in blue. However, given the different distribution of the IODC pixels grid (blue lines), the fire, in accordance with its actual position in the pixels of the RSS image, will be detected in a different pixel of the IODC image. That is, the fire in the pixel identified by the blue circle (see Fig. 3, right) would be detected in one of the four pixels identified by the yellow color numbers. Thus, this approach allows, in principle, the improvement of the geolocalization of the actual fire. Similar multiangle (stereoscopic) view approaches have been proposed for other purposes [16].

The general approach adopted herein is composed of two steps.

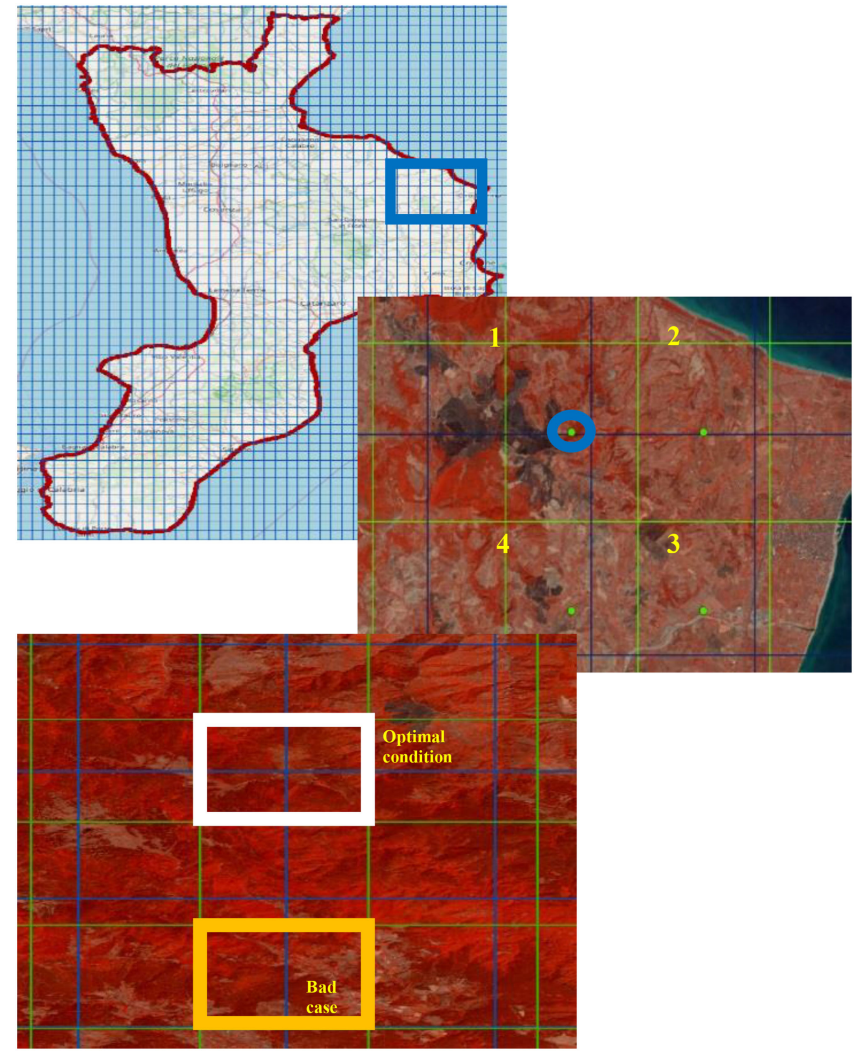

Fig. 3. Area of Interest. Top: the grid corresponding to the pixels of the SEVIRI/MSG-9 image of the Calabria region. Right: the difference in the grids distribution corresponding to MSG-9-RSS (green grid) and MSG-8-IODC (blue grid) images for the small area shown in the blue box. Bottom: an example of the relative positions of the pixels grid defining optimal and bad conditions for hotspot localization correction.

The first one aimed at coregistering the two satellite images at subpixel accuracy by following the methodologies developed in the last few years [17]-19]. Many applications require an image registration scheme to estimate the underlying correspondence between two or more images, which have been acquired either by a single sensor at different times, or by different sensors at the same time, but from different viewpoints, or by a combination of the above. Many techniques have been proposed for image registration. The most commonly used methods that provide subpixel accuracy are based on interpolation [18].

The technique proposed here is based on the evidence that a spatial domain technique is suitable for subpixel image registration [17]. The proposed technique aims at maximizing the correlation coefficient, which is a measure that provides robustness to photometric distortions. Most of the image registration algorithms are based on scale-invariant feature transform (SIFT) [19]. The geolocation of the fire within the pixel has been obtained by using an offset tracking approach based on subpixel cross-correlation between two simultaneously images acquired with different view geometry. The offsets due to the different viewpoints (atmospheric effect, pixel sizes, etc.) cannot be ignored. We will test the effect of these disturbances on the quality of the fire localization.

The method applied here is based on the normalized cross correlation which allows the derivation of a set of two-dimensional 
(2-D) offsets between reference image (RSS) and slave image (IODC). The similarity, defined as the correlation coefficient, is computed as follows [17]: Unnumbered eq. shown at the bottom of this page, where $\operatorname{Im}_{1}$ and $\mathrm{Im}_{2}$ represent the RSS and IODC images, respectively, with a 2-D offset $(\mathrm{a}, \mathrm{b})$ which can be described as: $\operatorname{Im}_{2}(x, y)=\operatorname{Im}_{1}(x-a, y-b) . \mathrm{M} * \mathrm{~N}$ is the correlation windows size. $\overline{I m_{1}}$ and. $\overline{I m_{2}}$ are the mean values of the pixels of the images pair in the correlation window. The NCC method searches for maximum correlation (i.e., maximum similarity) between window pairs formed by the two images. Those window pairs for which a maximum correlation is detected, are considered as corresponding pairs. From this, the 2-D offsets of the hotspot (fire) w.r.t. the coordinates of the pixel center can be obtained. To achieve the subpixel accuracy of correlation, the image intensities are oversampled prior to cross-correlation.

When the images are perfectly coregistered the combination of hotspots present on both of them allows the improvement of the geolocation of the events by exploiting the pixels grid intersection (see Fig. 3). The NCC method is applied to band 4 of SEVIRI images acquired at corresponding times by RSS and IODC, that is, every $15 \mathrm{~min}$.

\section{RESULTS AND DISCUSSION}

As can be deduced from Fig. 3, the improvement depends on the differences between the coordinates of the pixels in the two images. The maximum of the improvement can be obtained when the coordinates of the pixel in one image are at a distance of half pixel from the other ones (see Fig. 3). This can be explained by observing the right-bottom picture of Fig. 3 or, more clearly, the distribution of the pixel coordinates given in Fig. 4. As can be seen from Fig. 3, when the pixels coming from the two images are arranged as shown in the white box (optimal conditions), the fire, according to its actual position in the RSS/SEVIRI pixel, can occupy one of the four pixels of the IODC image. In the case shown in the orange box (bad case) of Fig. 3 the fire, according to its actual position, can be in one of two IODC image pixels. Fig. 4 shows that the mutual distance between pixel coordinates in the two observing systems presents a certain periodicity, which could be exploited to understand where this approach could be more useful. The possibility to improve the geolocalization of the hotspots was initially demonstrated by simulating the effect of a fire on pixels corresponding to RSS and IODC images. Then, it was validated by using satellite data.

\section{A. Case Study 1: Sicily Region}

One of the most interesting cases is the Sicily region for the huge number of fires that occur during the hot season.

As anticipated in the previous section, the RSS-IODC difference grid is periodic. Fig. 4 represents this first case study with

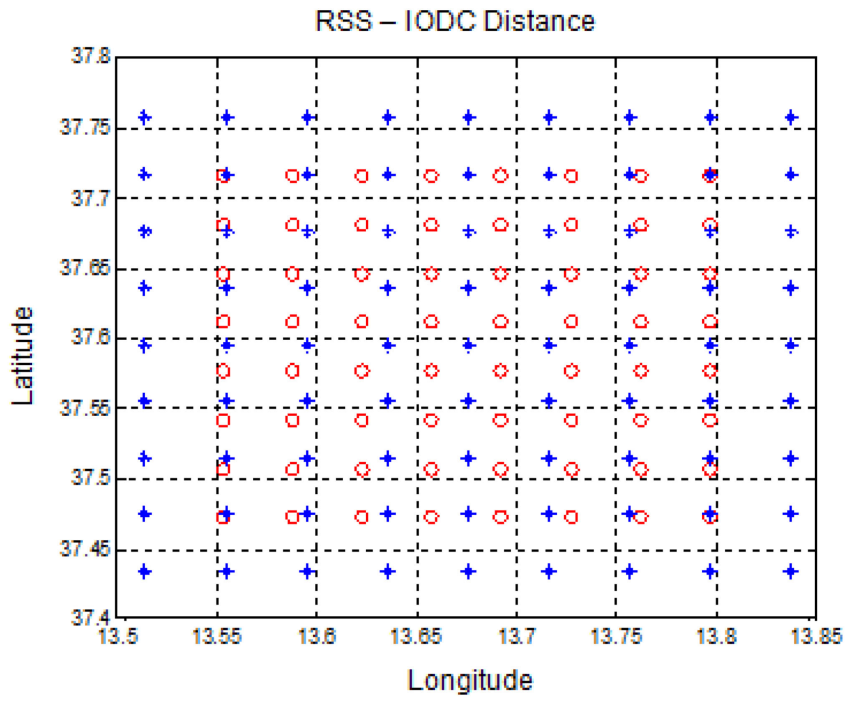

Fig. 4. Mutual lat-lon pixel coordinates of the RSS and IODC images. The distance between pixel-centers of the two images covering the same area shows a periodicity that, due to the different pixel size, is of $8 \times 8$ in the case of RSS (circles) and $7 \times 7$ in the IODC case (cross).

TABLE I

STATISTICS OF THE INTERPIXEL RSS-IODC DISTANCES IN THE CASE Study OF THE SiCILY REGion

\begin{tabular}{|c|c|c|c|}
\hline $\begin{array}{l}\text { Sicily Region } \\
\text { (5664 pixels) }\end{array}$ & $\begin{array}{l}\text { RSS-IODC } \\
\text { Interpixel } \\
\text { distance } \\
\left(\mathrm{d}<=0.01^{\circ}\right)\end{array}$ & $\begin{array}{l}\text { RSS-IODC } \\
\text { Interpixel } \\
\text { distance } \\
\left(0.01^{\circ}<\mathrm{d}<0.02^{\circ}\right)\end{array}$ & $\begin{array}{l}\text { RSS-IODC } \\
\text { Interpixel } \\
\text { distance } \\
\left(0.02^{\circ}<\mathrm{d}\right)\end{array}$ \\
\hline N. pixels & 1076 & 3224 & 1268 \\
\hline $\begin{array}{l}\% \text { Total } \\
\text { pixels }\end{array}$ & $19.32 \%$ & $57.90 \%$ & $22.78 \%$ \\
\hline Utility & Poor & Good & Excellent \\
\hline
\end{tabular}

the relative matrix of pixel coordinates differences, indicating, as the distance between pixel coordinates increases, what the hotspots present in the RSS image are for which the localization can be improved by using the corresponding IODC image. In contrast, hotspots occurring in the pixels with lower coordinates difference values would not benefit from this data integration.

From a statistical point of view, the results are shown in Table I. In this table, we divided the results into three possible cases: A case of poor utility with interpixel distance $\mathrm{d}<$ $1 \mathrm{~km}$ (second column); a good case with interpixel distance $1 \mathrm{~km}<\mathrm{d}<2 \mathrm{~km}$ (third column); and an excellent case with interpixel distance $\mathrm{d}>2 \mathrm{~km}$ (fourth column). The sum of columns 3 and 4, useful cases to improve the localization of hotspots, gives a result of 4492 useful pixels, that is, $80.67 \%$ of the total number of pixels covering the area (see Fig. 5).

$$
N C C=\frac{\sum_{i=1}^{M} \sum_{j=1}^{N}\left[\left(\operatorname{Im}_{1}(i, j)-\overline{I m_{1}}\right) \cdot\left(\operatorname{Im}_{2}(i, j)-\overline{I m_{2}}\right)\right]}{\sqrt{\sum_{i=1}^{M} \sum_{j=1}^{N}\left[\left(\operatorname{Im}_{1}(i, j)-\overline{I m_{1}}\right)^{2}\right.} \sqrt{\sum_{i=1}^{M} \sum_{j=1}^{N}\left[\left(I m_{2}(i, j)-\overline{I m_{2}}\right)^{2}\right.}}
$$



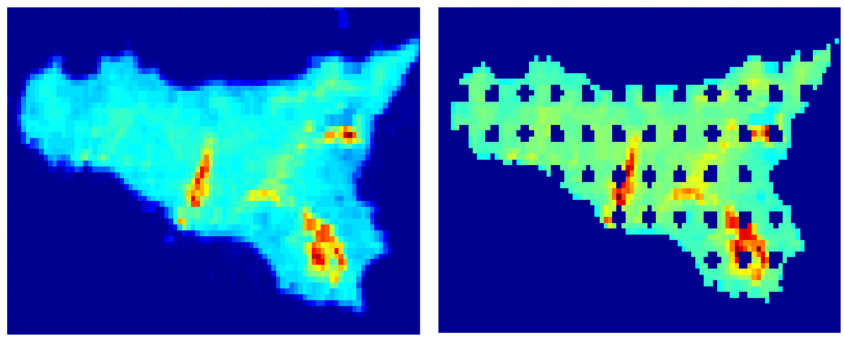

Fig. 5. Case study 1. Sicily Region (left) and the same territory (right) with the geographical areas (black zones) where there is no advantage in using the technique since $\mathrm{d}<1 \mathrm{~km}$. The higher the RSS-IODC differences $(\mathrm{d}>1 \mathrm{~km})$, the better the correction in the geolocalization of the hotspot will be.
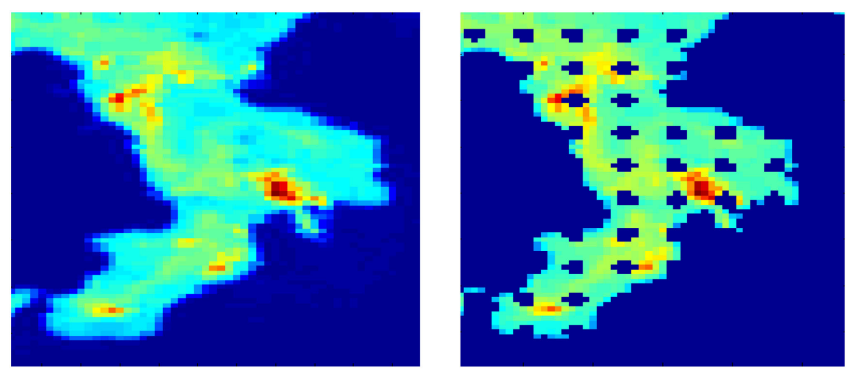

Fig. 6. Case study 2. Calabria Region (left). Calabrian territory (right) with the geographical areas (black zones) where there is no advantage in using the technique as $\mathrm{d}<1 \mathrm{~km}$. The higher the RSS-IODC differences $(\mathrm{d}>1 \mathrm{~km})$ the better the correction in the localization of the hotspot will be.

TABLE II

STATISTICS OF THE INTERPIXEL RSS-IODC DISTANCES IN the CASE Study of the CALAbria Region

\begin{tabular}{|c|c|c|c|}
\hline $\begin{array}{l}\text { Sardinia } \\
\text { Region } \\
\text { (4312 pixels) }\end{array}$ & $\begin{array}{l}\text { RSS-IODC } \\
\text { Interpixel } \\
\text { distance } \\
\left(\mathrm{d}<=0.01^{\circ}\right)\end{array}$ & $\begin{array}{l}\text { RSS-IODC } \\
\text { Interpixel } \\
\text { distance } \\
\left(0.01^{\circ}<\mathrm{d}<0.02^{\circ}\right)\end{array}$ & $\begin{array}{l}\text { RSS-IODC } \\
\text { Interpixel } \\
\text { distance } \\
\left(0.02^{\circ}<\text { d) }\right.\end{array}$ \\
\hline N. pixels & 825 & 2519 & 968 \\
\hline $\begin{array}{l}\text { \% Total } \\
\text { pixels }\end{array}$ & $19.13 \%$ & $58.41 \%$ & $22.46 \%$ \\
\hline Utility & Poor & Good & Excellent \\
\hline
\end{tabular}

\section{B. Case Study 2: Calabria Region}

Another case of extreme importance for the number of events that occur during the fire season is the Calabria region.

Even in this case study, the RSS-IODC difference grid is periodic. Fig. 6 represents this second case study with the relative matrix of differences. Such matrix indicates, as the value increases, what the hotspots present in the RSS image are for which the localization can be improved by integration with the corresponding IODC data. In contrast, hotspots occurring in pixels with lower values would not benefit from this data integration.

From a statistical point of view, the results are shown in Table II. In this table, as earlier, we divided the results into three possible cases: A case of poor utility with interpixel distance $\mathrm{d}<$ $1 \mathrm{~km}$ (second column), a good case with interpixel distance $1 \mathrm{~km}$ $<\mathrm{d}<2 \mathrm{~km}$ (third column); and an excellent case with interpixel distance $\mathrm{d}>2 \mathrm{~km}$ (fourth column). The sum of columns 3 and
4, useful cases to improve the localization of hotspots, gives a result of 3487 useful pixels, that is $80.86 \%$ of the total possible cases.

Figs. 5 and 6 show graphically data of Tables I and II. In fact, the blue areas present in the Sicily and Calabria regions represent the geographical areas where the technique here presented does not provide any improvement as the interpixel distance (d) is lower than $0.01^{\circ}$. The total area of the regions depicted in blue [in Figs. 5 and 6] is less than $20 \%$ in both cases [Tables I and II], demonstrating the potential of this technique. The theoretical analysis carried out above allows the assessment of the area of interest fraction for which a significant improvement of the hotspot geolocation can be expected. The following paragraph is devoted to demonstrating the improvement achievable by considering the actual events occurred during the 2017 fire season. The analysis has been extended to the entire Italian country in order to have a sufficient number of events to carry out a significant statistical analysis. At this stage, in order to simplify the analysis, the comparison has been made by using the hotspots provided directly by EUMETSAT based on the images acquired by MSG-HRIT and MSG-IODC, that is, the $15 \mathrm{~min}$ frequency images acquired by the two satellites located at $0^{\circ}$ and $41.5^{\circ}$ longitude, respectively. The improvement in the accuracy of the geolocation of the wildfires could be, in principle, assessed by using the following data and procedure.

1) Burned areas detected from high spatial resolution satellite images.

2) Fire locations provided by Italian National Fire Brigades.

3) Hotspots detected by using MODIS/VIIRS images as downloaded from firms.modaps.eosdis.nasa.gov.

4) Averaged differences between MSG HRIT single imagebased forest fire geolocation and above listed data.

5) Averaged differences between IODC corrected forest fire geolocation and earlier listed data.

To assess the improvement obtained in hotspots geolocation, the distribution of the relative pixels' distance should be taken into account.

Unfortunately, as explained by National Fire Brigades personnel, the coordinates of the fires in the reports they provide to us are not very accurate because, often, only the municipality in whose territory the fire occurred is recorded. On the other hand, the EUMETSAT fire detection methodology does not allow the detection of small fires and then in many cases the burned areas of the detected fires are too big to allow assessing the improvement in the geolocation accuracy (see Fig. 7). Further, the number of events was not statistically significant enough to perform an analysis of the accuracy improvement. Therefore, we decided to carry out the analysis by comparing the geolocation of MSG (EUMETSAT HRIT and IODC) hotspots [14], [15] with those provided by USGS based on MODIS [20], [21] and VIIRS [22] images, taken from FIRMS.

Fig. 8 shows the distribution of the fires detected in the whole of Italy from June 1 to September 30, 2017.

The analysis is based on the comparison of 33512 MODIS/VIIRS hotspots and 156320 MSG hotspots. It is worthwhile to note that the number of MODIS/VIIRS hotspots, if we consider their spatial distribution, is apparently larger than 

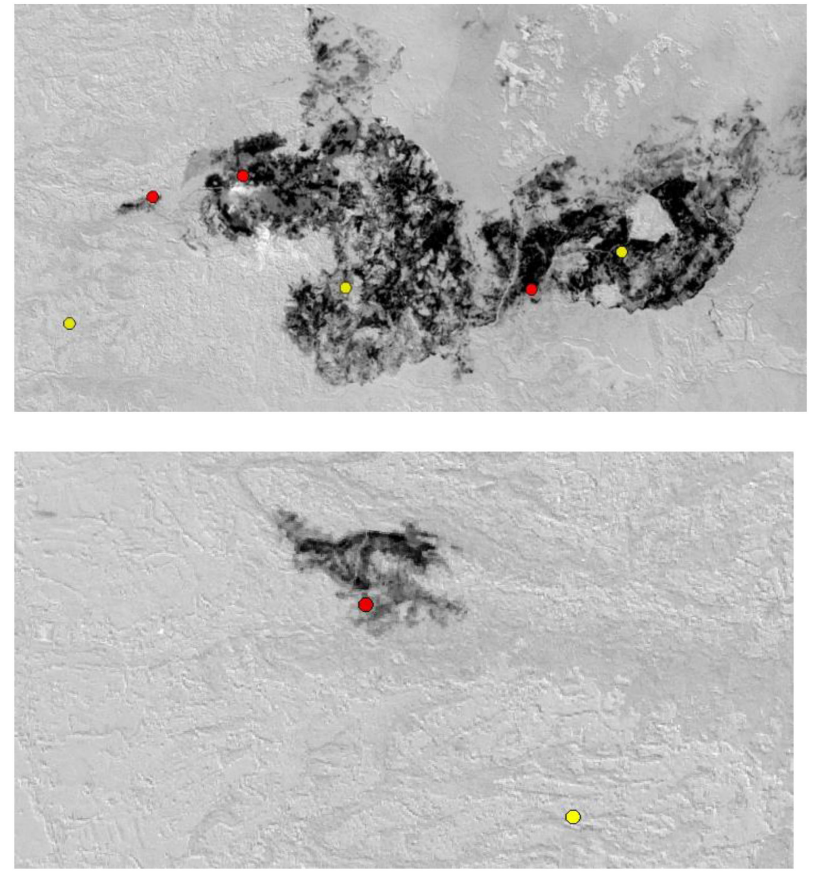

Fig. 7. Upper: Example of a large burned area (dark grey region) too extended to allow assessing any improvement in the geolocation of the fire with the technique proposed in the paper. In this case, the involved area covers more than $20 \mathrm{~km}^{2}$. Red dots correspond to the positions of the hotspots after the correction. Yellow dots represent the positions of the hotspots as detected by MSG-HRIT. Lower: Example of a small burned area for which the improvement of the hotspot geolocation is significant.

MSG (see Fig. 8) even if the numbers given above suggest the opposite. This is just because of the following reasons.

1) MODIS/VIIRS are able to detect smaller fires. Small wild fires affect all Italian regions.

2) MSG, due to its refresh frequency, is capable of detecting the same fire many times.

The analysis has been designed as follows.

1) For each MSG-HRIT hotspot the corresponding IODC hotspot is identified.

2) The HRIT coordinates of the hotspot are corrected by considering the overlapping area between the HRIT and IODC pixels in which the same hotspot is present.

3) The number of MSG hotspots for which a corresponding MODIS/VIIRS hotspot exists has been computed considering the hotspot coordinates before correction.

4) The number of MSG hotspots for which a corresponding MODIS/VIIRS hotspot exists has been computed considering the corrected hotspot coordinates.

Then, we check if the correction is capable of improving the correspondence between MODIS and MSG hotspots. In fact, we assume that the geolocalization is improved because MODIS geolocation uncertainty, given its better spatial resolution, could be considered lower.

The procedure has been implemented in MATLAB. Fig. 9 shows an example of overlapping between an MSG-HRIT and an MSG-IODC pixel in which the same hotspot has been detected. The actual position of the fire should occupy a point

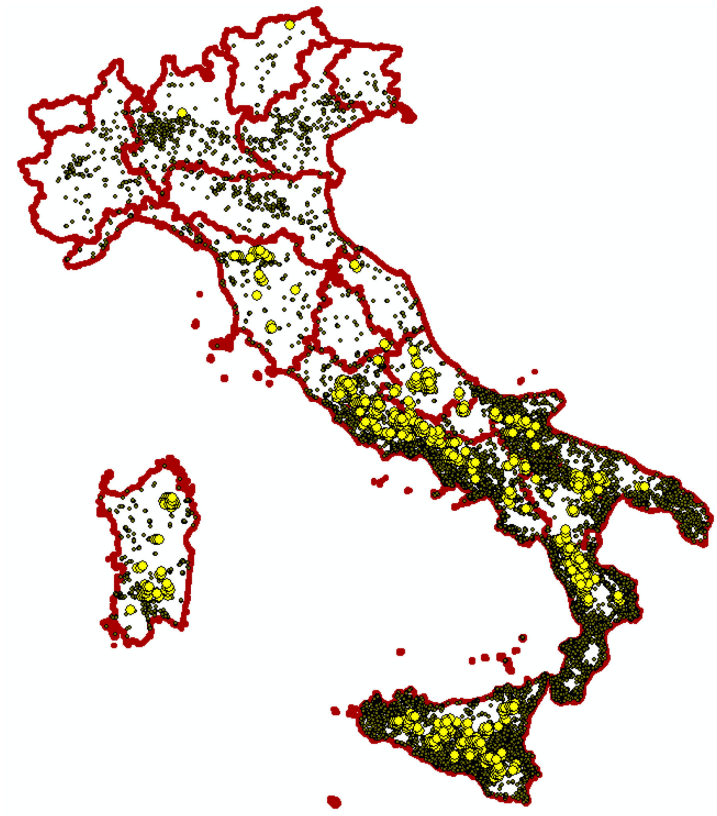

Fig. 8. Distribution of the fires detected by MODIS and VIIRS (green points, source USGS) and by MSG-HRIT (yellow dots, source EUMETSAT) from June 1 to September 30, 2017.

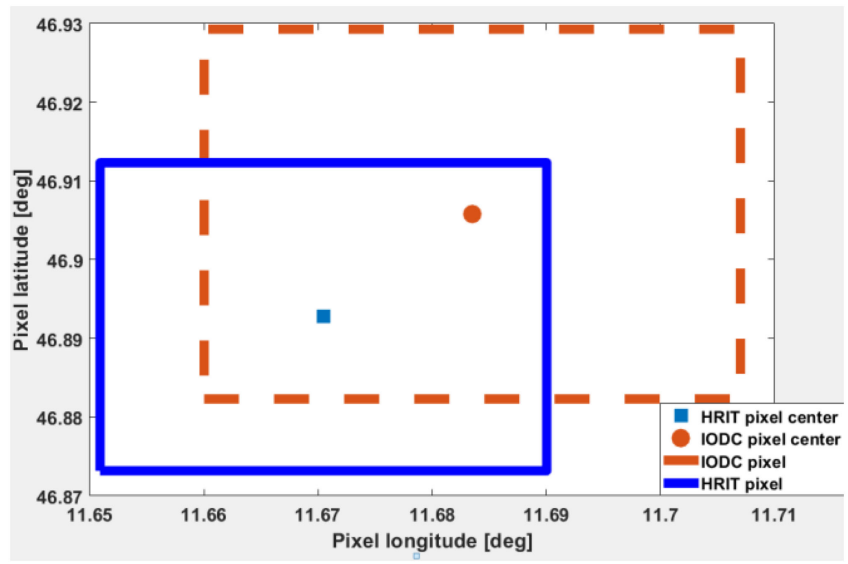

Fig. 9. Example of overlapping between a MSG HRIT and a MSG IODC pixel containing the same hotspot.

TABLE III

NUMBER OF MODIS FIRES FOR WHICH A MSG Fire HAS BEEN DETECTED AT A Distance LOWER THAN $2.5 \mathrm{KM}$

\begin{tabular}{lcc}
\hline \hline & HRIT & $\begin{array}{c}\text { HRIT-IODC } \\
\text { correction }\end{array}$ \\
$\begin{array}{l}\text { N. of MODIS/VIIRS fires at less } \\
\text { than 2.5 km from a MSG fire } \\
\text { \% Total fires }\end{array}$ & 22485 & 26615 \\
\hline \hline
\end{tabular}

within the overlapping area that is smaller than the area of the single pixel.

The results are synthesized in Table III. In particular, for $67 \%$ of MODIS hotspots there is at least a MSG HRIT hotspot falling into a circle of a radius of $2.5 \mathrm{~km}$ from the position of the MODIS hotspot. On the other hand, when we consider the hotspots with 


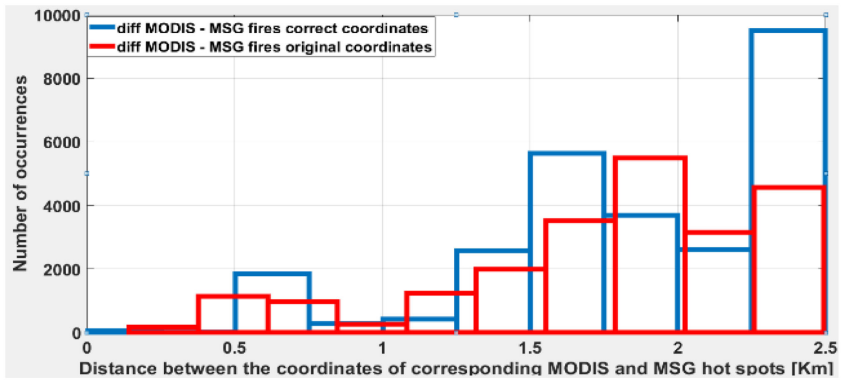

Fig. 10. Histogram of the distances between corresponding MODIS/VIIRS and MSG fires computed by using the original coordinates (red rectangles) and the corrected ones (blue rectangles).
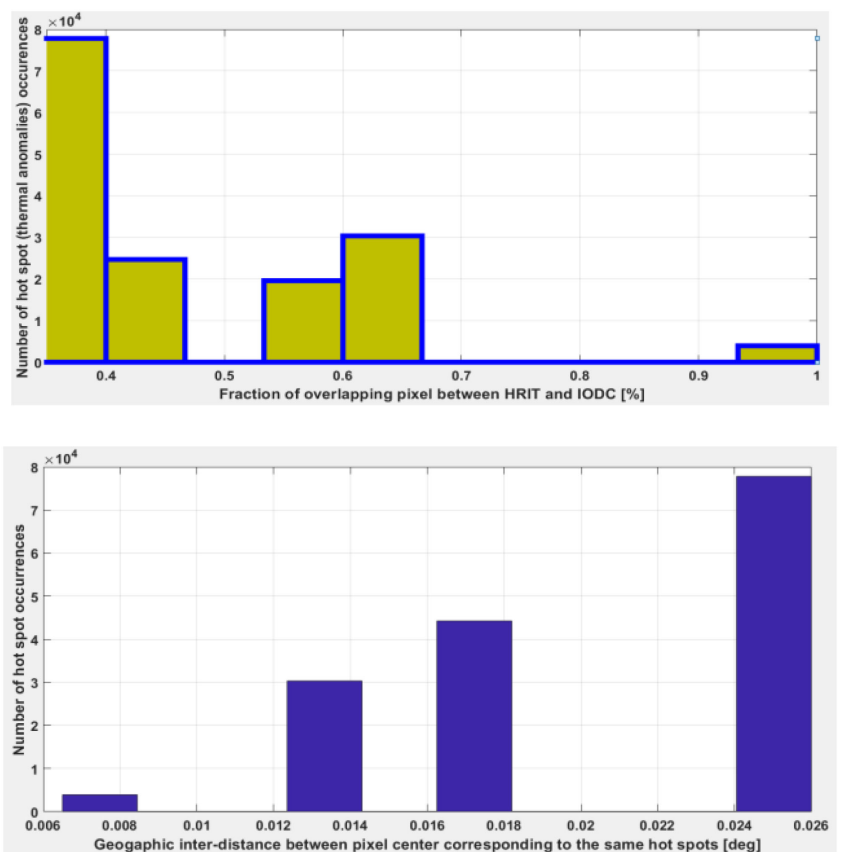

Fig. 11. Upper: Histogram of the overlapping fraction between HRIT and IODC pixels corresponding to the same hotspot. In most cases this fraction is lower than $25 \%$, resulting in an improvement in the fire geolocation from about $2 \mathrm{~km}$ to about $1 \mathrm{~km}$. Lower: Histogram of the geographic distance between HRIT and IODC pixels corresponding to the same hotspot. In about 50\% of cases the relative distance corresponds to the case defined as excellent in Tables I and II. That is, the distance is larger than $0.02^{\circ}$ (or $2 \mathrm{~km}$ ) and the geographic correction is as good as possible. In another $45 \%$ of cases such a distance is between 0.01 $(1 \mathrm{~km})$ and $0.02(2 \mathrm{~km})$, corresponding to the case good.

the position corrected by using those detected by MSG-IODC this percentage increases to $79 \%$. This confirms that, in general, using the information coming from both geostationary satellites could effectively increase accuracy in wildfire geolocation. The radius of $2.5 \mathrm{~km}$ has been selected taking into account that MODIS has a spatial resolution of $1 \mathrm{~km}$ and MSG of $4 \mathrm{~km}$, at Italian latitudes. Therefore the total uncertainty could be of $2.5 \mathrm{~km}$. Figs. 9 and 10 illustrate the results.

In particular, Fig. 10 shows the histogram of the distances between corresponding MODIS/VIIRS and MSG-HRIT hotspots, in $\mathrm{km}$, considering the original coordinates and those corrected by considering the MSG-IODC hotspot data.

Fig. 11 shows the following statement:
1) (Fig. 11, upper) the percentage of overlapping between MSG HRIT and IODC pixels affected by fire. In most cases, this overlapping is lower than $25 \%$ resulting in an improvement in the fire geolocation from about $2 \mathrm{~km}$ to about $1 \mathrm{~km}$.

2) (Fig. 11, lower) the geographic inter-distance between HRIT and IODC pixels corresponding to the same hotspot, in degrees. In about $50 \%$ of cases such a distance corresponds to the case defined as excellent in Tables I and II. That is, the distance is larger than $0.02^{\circ}$ (about $2 \mathrm{~km}$ ) and the geographic correction is as good as possible. In another $45 \%$ of cases such a distance is between 0.01 $(1 \mathrm{~km})$ and $0.02(2 \mathrm{~km})$, corresponding to the case defined as good in Tables I and II.

\section{CONCLUSION}

One of the objections to the idea of using sensors on board of geostationary satellites to monitor forest fires in a quasicontinuous way concerns the rough spatial resolution of these systems, not permitting to achieve the geolocalization accuracy of the event required by operational applications. This paper aims at analyzing the possibility of overcoming, at least partially, this limit by using data collected simultaneously, from two different view angles, from two geostationary satellites located at different longitudes. The noncoincident grid of pixels of the two images can be exploited to reduce the uncertainty concerning the geolocation of the hotspot. However, since the correspondence between the pixel grids of the two simultaneous images is not constant along the image, the possibility of improving the fire event geolocation is not the same everywhere. Anyway, the method shows that, considering two particular areas (Sicily and Calabria), the percentage of favorable cases (with an interpixel distance RSS-IODC $>1 \mathrm{~km}$ ), is more than $80 \%$ of the potential events.

The possibility of the method to effectively improve hotspot localization has been demonstrated by comparing the positions of the hotspots detected by MSG with those detected by MODIS. In fact, when the positions of the hotspots detected by MSG HRIT $\left(0^{\circ}\right.$ longitude) are corrected by considering the hotspots detected simultaneously by MSG IODC $\left(41.5^{\circ}\right)$, the number of MSG hotspots falling at a distance of $2.5 \mathrm{~km}$ from MODIS hotspots increases from $67 \%$ to $79 \%$.

Possible future developments concern the analysis of other Italian and European locations with a high forest fire risk, and the operational implementation of the method in our automatic processing chains for hotspot detection.

A further improvement of the geolocation of the hotspots detected by using geostationary satellites will be obtained by using a higher spatial resolution $(500 \mathrm{~m})$ vegetation fuel map of the area of interest. This will allow associating the hotspot position (corresponding to the pixel center coordinates) to a probability distribution function of the fire position, based on the characteristics of the vegetation in the area of reduced uncertainty, obtained by combining HRIT and IODC hotspots or RSS and IODC hotspots. 


\section{ACKNOWLEDGMENT}

The authors would like to thank VVFF for providing the list of forest fire events that occurred in Italy in the year 2017.

\section{REFERENCES}

[1] JRC Report n.10, "Forest fires in Europe 2009," EUR 24502 EN, 2010.

[2] S. P. Flasse and P. A. Ceccato, "A contextual algorithm for AVHRR fire detection," Int. J. Remote Sens., vol. 17, no. 2, pp. 419-424, 1996.

[3] C. O. Justice et al., "The MODIS fire products," Remote Sens. Environ., vol. 83, no. 1/2, pp. 244-262, Nov. 2002.

[4] E. M. Prince and W. P. Menzel, "Geostationary satellite detection of biomass burning in South America," Int. J. Remote Sens., vol. 13, no. 15, pp. 2783-2799, 1992.

[5] C. Justice, J. P. Malingreau, and A. W. Setzer, "Remote sensing of fires: Potential and limitations," in Fire in the Environment: The Ecological, Atmospheric, and Climatic Importance of Vegetation Fires, P. J. Crutzen and J. G. Goldammer, Eds. Hoboken, NJ, USA: Wiley, 1993, pp. 7788. [Online]. http://queimadas.cptec.inpe.br/ rqueimadas/documentos/ 1993_Justice_Malingreau_Setzer_Satellite_Dahlem.pdf

[6] Z. Li et al., "A review of AVHRR-based active fire detection algorithms: Principles, limitations, and recommendations," in Global and Regional Vegetation Fire Monitoring From Space: Planning a Coordinated International Effort. The Hague, The Netherlands: SPB Academic, 2001, pp. 199-225.

[7] J. M. Robinson, "Fire from space: Global fire evaluation using infrared remote sensing," Int. J. Remote Sens., vol. 12, no. 1, pp. 3-24, 1991.

[8] A. W. Setzer and J. P. Malingreau, "AVHRR monitoring of vegetation fires in the tropics: Toward the development of a global product," in Biomass Burning and Global Change, J. S. Levine, Ed. Cambridge, MA, USA: MIT Press, 1996, vol. 1, pp. 25-39.

[9] Y. Li, A. Vodacek, R. L. Kremens, A. Ononye, and C. Tang, "A hybrid contextual approach to wildland fire detection using multispectral imagery," IEEE Trans. Geosci. Remote Sens., vol. 43, no. 9, pp. 2115-2126, Sep. 2005.

[10] A. Calle, J. L. Casanova, and A. Romo, "Fire detection and monitoring using MSG spinning enhanced visible and infrared imager (SEVIRI) data," J. Geophys. Res., vol. 111, 2006, pp. 1-13.

[11] C. Filizzola et al., "RST-FIRES, an exportable algorithm for early-fire detection and monitoring: Description, implementation, and field validation in the case of the MSG-SEVIRI sensor," Remote Sens. Environ., vol. 192, pp. e2-e25, 2017.

[12] G. Laneve, M. M. Castronuovo, and E. Cadau, "Continuous monitoring of forest fires in mediterranean area using MSG," IEEE Trans. Geosci. Remote Sens., vol. 44, no. 10, pp. 2761-2768, Oct. 2006.

[13] G. Laneve and V. Di Biase, "Geostationary sensor based forest fire detection and monitoring: An improved version of the SFIDE algorithm," Remote Sens. J., vol. 10, pp. 741-759, 2018, doi: 10.3390/rs10050741.

[14] "MSG meteorological products extraction facility algorithm specification document," EUMETSAT, Darmstadt, Germany, 2015, [Online]. Available: https://www.eumetsat.int/website/home/Data/TechnicalDocuments/ index.html

[15] Operations product validation report: FIR product, EUMETSAT, Darmstadt, Germany, 2009.

[16] L. Merucci, K. Zaksek, E. Carboni, and S. Corradini, "Stereoscopic estimation of volcanic ash cloud-top height from two geostationary satellites," Remote Sens., vol. 8, no. 3, pp. 2-19, 2016.

[17] L. Sun and J. P. Muller, "Evaluation of the use of sub-pixel offset tracking techniques to monitor landslides in densely vegetated steeply sloped areas," Remote Sens., vol. 8, no. 8, pp. 659-684, 2016.

[18] I. Karybaly, E. Psarakis, K. Berberidis, and G. Evangelidis, "An efficient spatial domain technique for subpixel image registration," in Signal Processing: Image Communication, Amsterdam, The Netherlands: Elsevier, 2008, vol. 23, no. 9, pp. 711-724.

[19] F. Dellinger et al., "SAR-SIFT: A SIFT-like algorithm for SAR images," IEEE Trans. Geosci. Remote Sens., vol. 53, no. 1, pp. 453-466, Jan. 2015.
[20] FIRMS, 2018. [Online]. Available: firms.modaps.eosdis.nasa.gov/

[21] C. O. Justice et al., "MODIS-derived global fire products," in Land Remote Sensing and Global Environmental Change. Remote Sensing and Digital Image Processing, B. Ramachandran, C. Justice, and M. Abrams, Eds. vol. 11, New York, NY, USA: Springer, 2011 pp. 661-679, doi: 10.1007/978-1-4419-6749-7_29.

[22] W. Schroeder, P. Oliva, L. Giglio, and I. A. Csiszar, "The New VIIRS $375 \mathrm{~m}$ active fire detection data product: Algorithm description and initial assessment," Remote Sens. Environ., vol. 143, pp. 85-96, 2014.

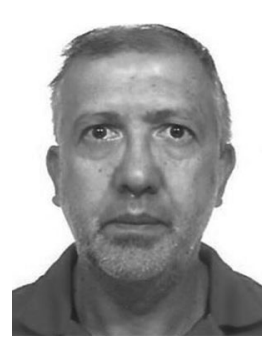

Giovanni Laneve (M'02) was born in Viggiano, Italy, in 1960. He received the Laurea degree in Aeronautical engineering from the Università di Napoli, Naples, Italy, in 1985, and the Laurea degree in aerospace engineering from the Sapienza University of Rome, Rome, Italy, in 1988.

From 1987 to 1991, he was a Consultant with the San Marco Project Research Centre, Rome, Italy, where he was involved in the San Marco 5 satellite mission control and data analysis. He is currently an Associate Professor with the School of Aerospace Engineering, Sapienza University of Rome. He has authored more than 160 scientific papers. Since 1988, he has been teaching the course of aerospace systems for remote sensing with the School of Aerospace Engineering. His research interests include aeronomy, satellite thermal control, mission design, new algorithms for the exploitation of satellite images, satellite remote sensing applications for fire management, applications of satellite data for the African regions, and studies on environmental and disaster monitoring.

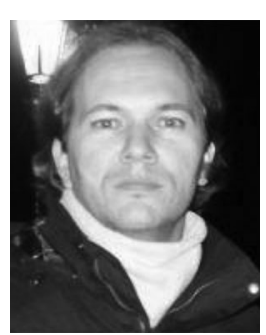

Giancarlo Santilli was born in Valera, Venezuela, in 1974. He received the Laurea degree in electronic engineering in 2000 from the Sapienza University of Rome, Italy, where he also received the Ph.D. degree in aerospace engineering from the Aerospace Engineering School in 2011.

From 2004 to 2013, he was a Consultant Researcher with the San Marco Project Research Centre (CRPSM), Rome, Italy. From 2013 to 2015, he was Consultant at Telespazio (TPZ), Rome, Italy. Since 2015, he has been an Associate Professor with the Gama Faculty (FGA) - University of Brasilia (UnB), teaching earth observation, orbital dynamics, and electronic engineering courses. He has authored more than 60 scientific papers. His research interests include remote sensing applications, mission design for EO, TT\&C activities, and developing of algorithms for the exploitation of satellite images.

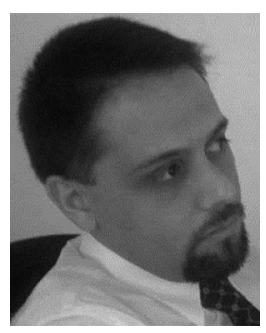

Roberto Luciani was born in Rome, Italy, in 1985. He received the B.Sc. degree in aerospace engineering, in 2010, the M.Sc. degree in astronautical engineering, in 2014, and the Ph.D. degree in energy and environment from the Sapienza University of Rome, Rome, Italy, in 2018.

His master's thesis and research involves applying remote sensing, image processing, and spatial analysis to assess the impact of natural and anthropogenic stressors on the marine and terrestrial ecosystems. 